\title{
Quaternary quality a medium quality that connects macro quality with micro quality
}

\author{
Mingshun Song ${ }^{1, *}$, Tingting $\mathrm{Zhu}^{1}$, and Binfang $\mathrm{Qi}^{1}$ \\ ${ }^{1}$ China JiLiang University, Hangzhou, Zhejiang, China
}

\begin{abstract}
To construct the outlook on great quality, the concept of quality can be divided into two aspects, micro quality and macro quality at present. However, the definition of micro quality and its contents are not related organically with the conception of macro quality and its contents. So an intermediate variable between micro quality and macro quality is needed to link them together, and this intermediate variable is named as medium quality. This paper introduces the connotation of medium quality that includes the concept of quaternary quality, namely zero quality, unary quality, binary quality and ternary quality, and explains the progressive logical relationships of these four qualities. Micro quality and macro quality are cohered by medium quality organically, and thus formulates the scientific outlook on great quality.
\end{abstract}

\section{Introduction}

Nowadays many countries attach great importance to the quality of economic development, which is defined as development quality in this paper. Therefore, one of the most important goals for China to advance along the path of scientific development is the pursuit of development quality. Development quality, which consists of core factors including reputation, market rules, market competitiveness and brand, is the degree for economic development to meet the requirements of the whole society and sustainable development. Development quality, also known as macro quality, is one of the hottest topics in quality academia in recent years ${ }^{[1]}$. Product quality, which includes hardware product quality, software product quality, processed material quality, service quality and project quality, is the traditional research field in academia. Relative to development quality, product quality is also called micro quality. The concept of quality is divided into micro and macro aspects, and thus formulates the outlook on great quality ${ }^{[2]}$. According to the research paradigm, micro quality and macro quality shall have a logical relationship, but now this relationship is ambiguous. Quality practitioners and academia still focus on the concept of product quality, and the definition of product quality mainly based on the International Organization for Standardization (ISO), that is, "Degree to which a set of inherent characteristics fulfils requirements." This definition can be interpreted as the degree that product characteristics conform to standards and meet the requirements of customers ${ }^{[3]}$. The formation of the outlook on great quality must be based on the combination of micro quality and macro

\footnotetext{
Corresponding author: $\underline{\text { smsqm@,cjlu.edu.cn }}$
} 
quality, that is, macro quality embeds micro quality, and micro quality determines macro quality. An intermediate variable between micro and macro quality is needed to link them together, and this intermediate variable is named as medium quality. The connotation of medium quality must be able to explain the logical relationship between micro quality and macro quality, thus the quality concept of ISO is extended from a new perspective, so as to construct a scientific outlook on great quality. To this end, the paper puts forward the concept of quaternary quality, and constitutes the connotation of medium quality in accordance. It can link micro quality with macro quality organically, and make up the scientific outlook on great quality simultaneously. (As shown in fig. 1)

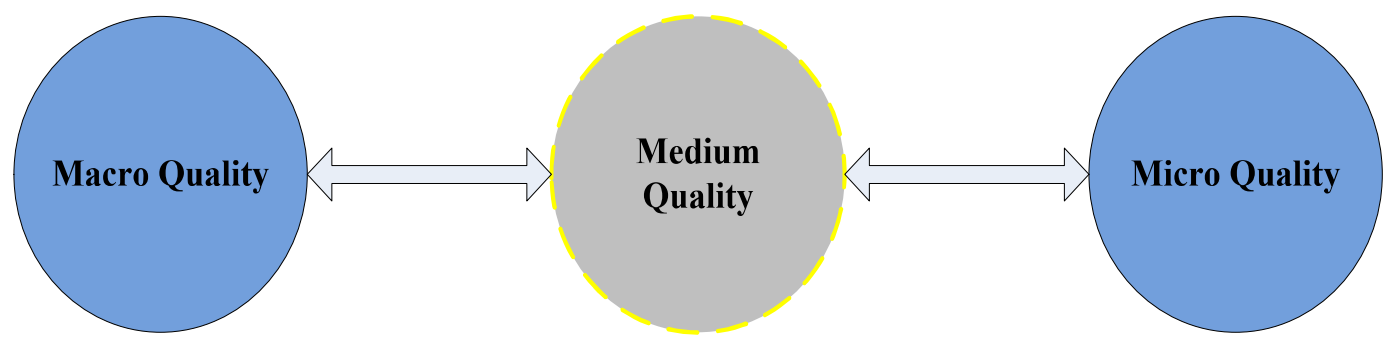

Fig. 1. The framework of the outlook on great quality

\section{Literature review}

Medium quality is explained by quaternary quality that can be described from four dimensions, that is, zero quality, unary quality, binary quality and ternary quality. Zero quality can be named as moral quality; unary quality also be referred to conformity quality; binary quality can be referred to applicability quality; ternary quality also be named as excellence quality. If regard the medium quality as a quality equation, the equation has four variables, that is to say, the equation is a quaternion equation which consists of zero quality, unary quality, binary quality and ternary quality.

\subsection{The Connotation of Zero Quality}

Zero quality, which is also named as moral quality, refers to the degree that products meet social ethics and public morality. Zero quality actually reflects the level of "quality faith" of enterprises. The integration of the levels of zero quality in all enterprises reflects the situation of "quality faith" in a region or a country. Some poor zero quality problems have turned up both in domestic and abroad frequently. At the beginning of the last century, Chicago slaughterhouse made sausage with dead pork, moldy pork and poisoned mice, and sold them both in domestic and international market. The incident not only brought about an extreme panic for domestic consumers, but also made international market give rise to an all-round blockade to US food, causing a half-decadent situation for US livestock. Due to the serious zero quality problems, the US food production caused country's credit crisis ${ }^{[4]}$. Another case is the Toyota recalls. The global outbreak of Toyota recalls in early 2010 directly exposed zero quality problems. Due to the devotion of exclusive attention to output, Toyota Motor Corporation ignored its quality and turned up some serious quality problems, such as power steering failure and engine failure, causing many injuries and deaths of people, as well as property damages. Because of the poor zero quality problems, Toyota had been recalled nearly 8.5 million vehicles, which made the credit of Toyota suffer an unprecedented blow, and also made a negative impact on Japan's economy ${ }^{[5]}$. For reducing the cost, China Shijiazhuang Sanlu Group co., LTD., once a famous Chinese dairy products enterprise, added industrial "melamine" into the infant milk powder to improve 
the protein content, causing serious kidneys damage of children who had ate it. The enterprise had much poor zero quality problems, and eventually made itself bankruptcy.

The quality culture undertook by enterprises and societies which take the values as the core and the moral spirit as the soul is the base to boost the level of zero quality. Carrying out all kinds of corporate social responsibility standards and certifications are the most important means for enterprises to enhance the level of zero quality. Improving the quality regulations of government is the external security to increase the level of zero quality. The enterprises that have zero quality problems have survival danger, so zero quality is their life. Enterprises whose products and business are closely related to people's life and property, animals and plants' health, should pay more attention to zero quality, and should focus their attention on the construction of quality culture and risk prevention mechanism, and also the education of risk consciousness.

\subsection{The Connotation of Unary Quality}

Unary quality, which is also named as conformity quality, refers to the degree that products comply with relevant quality standards. In order to judge the degree of meeting the requirements of standards, enterprises shall organize production based on the relevant standards, and products should be tested by the related inspection instruments. Unary quality is a kind of objective quality. Philip B. Crosby defined quality as "conformity quality", and believed that quality was tangible and also could be measured ${ }^{[6]}$. The level of unary quality actually reflects the level of standards, that is to say, high level of standards maybe achieve high level of unary quality. Unary quality usually be described with conformity and no conformity. If the products produced by enterprises and commodities circulated in the market have certification marks, it proves that the products and commodities conform to the relevant standards. Most enterprises have quality inspection departments to be in charge of testing the unary quality of their products. Both of the supervision on product quality and the testing on imported goods are the methods to judge the level of unary quality by governments. The product certifications engaged by various quality certification bodies are the documentary evidences of products to meet the unary quality. The CCC (China Compulsory Certification) mark printed on the product or its package means the product meets the safety standards. Up to March 2012, the number of CCC certification has grown to 22 major categories and 163 kinds of products, covering 10 industries that related to human health and safety, and have important influence on national economy. Nearly 618,000 CCC certificates licenses had been issued to 88,000 enterprises ${ }^{[7]}$. That is to say, nearly 618,000 kinds of products had met the requirement of unary quality.

Quality inspection is one of the main methods for unary quality management. Furthermore, standards, testing equipment and laboratory facilities are the most important means to improve the unary quality. The level of the technical standards determines the level of unary quality directly, and testing level determines the reliable level of testing results for unary quality. The main method to improve the testing level is standardization. For example, implementing ISO 10012-1 and ISO 10012-2 standards can assure the measuring reliability of inspection departments of enterprises. To engage in product quality certification, testing and calibration laboratories must carry on ISO/IEC 17025 standard, and gain the laboratory accreditation. Sampling technology is also an important tool to assure the unary quality. Most of the quality inspections are sampling inspection. The rationality of sampling schemes determines the reliability of unary quality directly. There are many sampling standards for sampling inspection. For the enterprises that produce spare parts and semi-finished products, outsourcing services, or the enterprises that produce products based on order forms, or the enterprises that are in the middle and lower positions 
of the supply chain, unary quality is their life, and conforming to the standards is their highest goals.

\subsection{The Connotation of Binary Quality}

Binary quality, which is also named as applicability quality, refers to the degree that products meet the requirements of customers or market. When the product standards are not adapted to the market requirements, even if the product quality achieves the high level of unary quality, the products also have no market share and no benefits, because the product quality doesn't meet the requirements of customers. While meeting the requirements of the standards, many products are surplus and overstocked because of the dissatisfying with market requirements. For instance, Global Satellite Mobile Communication System, which was invented by American Iridium Satellite Company, was unable to form the stable customers bases because of its quality problems, such as terminal bulky, capacity insufficient, communication prolonged, lower spectrum utilization, and the failure to provide the personal mobile communication business based on the handsets. Furthermore, its standards lag behind the requirements of the market ${ }^{[8]}$, so the enterprise officially declared bankruptcy on March 17, 1999. For another instance, the mobile phones produced by Nokia and Ericsson companies once became the main supply products in the market because of using the standard of second generation mobile communication. But when the smart-phone appeared, they lost the mobile phone market and were replaced by Apple and Samsung. Nokia and Ericsson companies failed to create applicable products in the light of new standards, so their product quality stayed in the original unary quality and couldn't satisfy the new requirements of customers. Therefore, improving the level of binary quality and satisfying the requirements of customers are the foundation of sustainable development of the enterprises. There are lots of methods and means to improve the level of binary quality. Market research is a market-oriented way to improve the level of binary quality. It can find the actual and potential requirements of the customers. Then new standards are set based on these requirements, and thus the products that can satisfy these requirements are produced in terms of these standards. Innovation creates the market or drives the requirements, and these requirements are led by innovation achievements. The achievements of science and technology innovation create customers' requirements, which are the unexpected requirements for customers. Apple Inc. innovates the smart-phones by applying breakthrough technology, which created the market directly and brought the unexpected requirements for customers, so as to improve the level of binary quality. The construction of quality culture and the management of customers relationship which regard "Customers are always right" as the core principle are the effective methods to improve the binary quality of enterprises. The standard of ISO/TS10004:2010 quality management customer satisfaction monitor and measure guide is also an important mean to improve the binary quality. For the enterprises whose products sale to the terminal market directly or direct-to-consumer, binary quality is their life. Enhancing the level of binary quality is the core factor of quality managements for these enterprises.

\subsection{The Connotation of Ternary Quality}

Ternary quality, which is also named as excellence quality, refers to the degree that enterprises pursue the business excellence. The essence of ternary quality is the business quality of enterprises and the level of brand construction. Enterprises gain excess profits by low cost of investment and excellent management, so as to bring great value to customers. On the contrary, if enterprises only stay in binary quality level, they may be unable to obtain excess profits. There are many enterprises that may have good shares in the market, 
but their benefits are low, and even have no benefits. For example, the ties produced by enterprises that are located in Shengzhou county, Zhejiang province, had shared $60 \%$ of the international market, but the profits were less than $1 \%$. Because these products failed to become an international brand, although the level of binary quality of the enterprises that produced these products was very high, their benefits were low $^{[9]}$. For another example, the profit rate of Volkswagen in China was $11.3 \%$, but the rate of domestic automobiles was merely less than $3 \%^{[10]}$. The reason is very simple: Volkswagen is a famous international brand, but our car manufacturers are not. Quality is the core factor of building a brand, and the path of building a brand is the pursuit of excellence business to enterprises. There are many methods to improve the ternary quality, such as marketing, advertising etc. It turns out that the Six Sigma management and Criteria for Performance Excellence are ones of the most effective and widely used methods for enterprises to improve ternary quality, pursue excellence business, and build a brand. No matter what kinds of enterprises, only if they have the pursuit of lasting forever and sustainable development mode, the high level of ternary quality is their essential foundation.

\section{Logical Relationships}

Unary quality, binary quality and ternary quality have a progressive logical relationship, that is, if enterprises want to pursue ternary quality, they must satisfy the requirements of unary quality and binary quality simultaneously. Zero quality is the foundation of all the other three qualities. When zero quality is poor, the quality tower will collapse, and everything will return to zero. Therefore, the structure of medium quality is a pyramid, which can also be named as quality pyramid. (As shown in fig. 2)

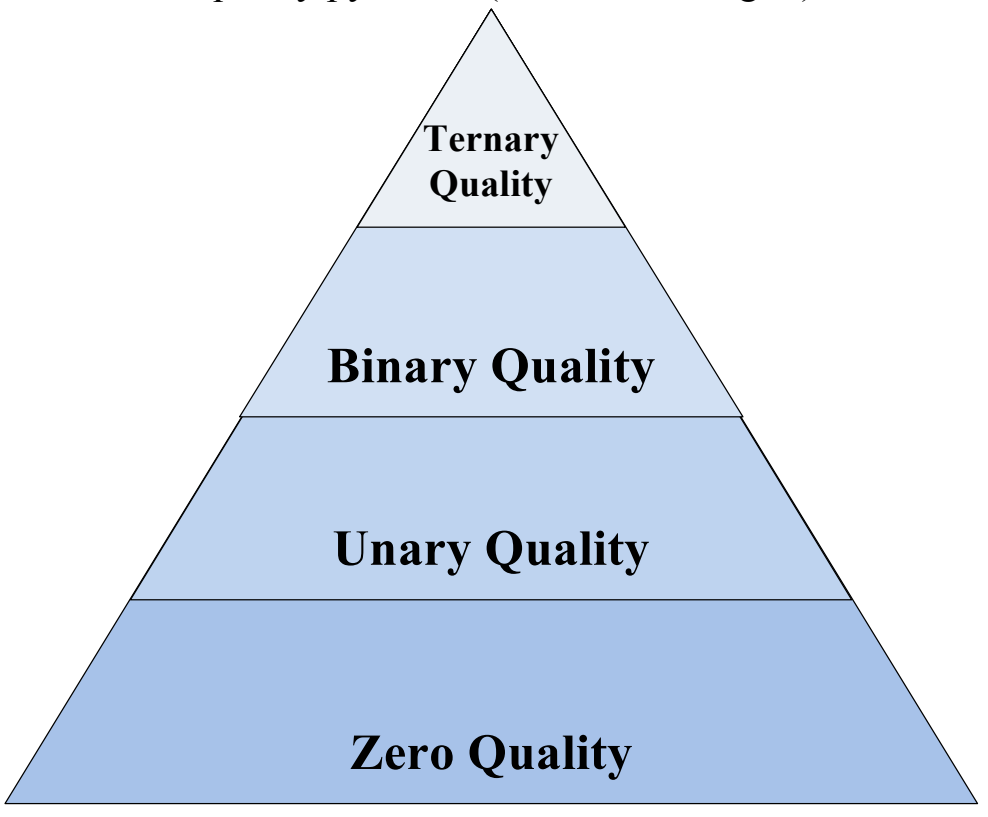

Fig. 2. Quality pyramid.

Quality pyramid links micro quality and macro quality together. The core elements of macro quality are credit, voice in standards, market share and the number of international brands. For a country or a region, the level of credit depends on the quality faith, and zero quality is the foundation of building quality faith. Voice in standards, which mainly refers to the voice in the international market, represents the influence on the country or region. If the standards are controlled, then the market will be controlled. The high level of unary quality is the most important way to promote the standardization construction. Only high standards can bring about high quality, and the high standards depend on a high level of 
scientific and technological innovation, while the technological innovation can drive economic development. The international market share is closely related to the international market competitiveness, and only the pursuit of a high level of binary quality can lead to a high level of market share. The amounts of international brands affect the core competitiveness of a country or a region directly, and also determine their development level. For the annually top 500 of international brands ranked by World Brand Laboratory, the number of brands in United States, Japan, Britain, France and Germany have been always top five ${ }^{[11]}$. These five countries also have been the highest level of development quality in the world. Therefore, the pursuit of excellence business and building brands are ones of the most effective means to improve the ternary quality.

\section{The Outlook on Great Quality}

Medium quality, which makes quaternary quality as the core factor, combines micro quality with macro quality naturally and organically, then the outlook on great quality can be formed accordingly. The outlook on great quality shows that the development quality of economy in a country or a region depends on the quality of products. Only promote the level of product quality down-to-earth can we improve the development quality of economy, and the economic development processes of United States, Japan and Germany have proved this regular pattern. These three countries have the highest level of macro quality in the world, because they have the highest level of micro quality. Their micro qualities also have distinguishing features: the quality feature of United States is innovation; the quality feature of Japan is lean and the quality feature of Germany is reliability. Therefore, the outlook on great quality shows that only a high level of product quality can assure the development quality of national or regional economy. Improving the level of quality management of the enterprises and the level of quality governance of a society are the foundation to enhance the development quality of economy.

\section{References}

1. C. Hong. Journal of Wuhan University,129-134(2010)

2. Lang Zhizheng. "The Outlook on Great Quality That I Had Seen." Supervision and Selection (November): 4(2002)

3. S. Mingshun. Quality Management, second edition. Beijing: Science Press (2012).

4. U. Sinclair. The Jungle. Si Chuan: Sichuan Literature and Art Press (2010).

5. X. Guojun. “'Toyota Recalls' is a Game about what?” China equipment (April): 90 (2010).

6. P. Crosby. Quality Is Free The Art of Making Quality Certain. Beijing: China Renmin University Press (2006).

7. S. Jingjing."Perfecting Compulsory Product Certification System in China." (April): 14(2012)

8. L. Lichun. Telecommunications Science, 31(1999).

9. L. Yabiao and S. Yiying. "Shengzhou Tie : Aphasia of The World's Largest." Economic Information Daily (2010)

10. Fortune. The World Fortune Rankings of Top 500 in 2013 [ EB/OL ] http://money.cnn.com/magazines/fortune/rankings/?iid=F_Sub(2013). 
11. World Brand Laboratory. The World Brand Rankings of Top 500 in 2012 [ EB/OL ] : http://www.worldbrandlab.com/world/2012/ 